\title{
Iterative geometric triangle transformations
}

Dimitris Vartziotis and Simon Huggenberger

\begin{abstract}
Dimitris Vartziotis studied aerospace engineering and civil engineering at the University of Stuttgart, Germany. He is head of the Mathematics Research Department at TWT GmbH Science \& Innovation, Stuttgart. Furthermore, he is currently researcher at the Institute of Structural Analysis \& Antiseismic Research at the National Technical University of Athens, Greece.

Simon Huggenberger studied mathematics at the University of Tübingen, Germany, and did his Ph.D. at the University of Ghent, Belgium, in the field of discrete geometry. Currently, he is a member of the Mathematics Research Department at TWT GmbH Science \& Innovation, Stuttgart.
\end{abstract}

\section{Introduction and notations}

A well-known theorem in geometry of triangles is the following:

If equilateral triangles are erected externally on the sides of any triangle, their centres form an equilateral triangle.

This theorem can be found in [3] and is often attributed to Napoleon Bonaparte, although it is questionable whether he knew enough geometry for this achievement, see [1].

There are several generalisations of this theorem. One is the following, which can be found in $[7,4$.$] or [1$, Theorem 3.36]:

Seit über einem Jahrhundert sind geometrische Transformationen von Dreiecken Gegenstand mathematischer Untersuchungen. In dem vorliegenden Artikel wird ein Ausgangsdreieck in ein neues Dreieck transformiert, indem auf jeder der Dreiecksseiten ein gleichschenkliges, nach außen gerichtetes Aufsatzdreieck errichtet wird. Die Scheitelpunkte der Aufsatzdreiecke bilden die Eckpunkte des neuen Dreiecks. Durch wiederholtes Anwenden dieser Transformation erhält man eine Folge von Dreiecken. Die Form der Dreiecke dieser Folge konvergiert gegen eine charakteristische Dreiecksform, von der nachgewiesen wird, dass sie mit jedem Transformationsschritt echt angenähert wird. 
If similar triangles $P C B, C Q A$, and $B A R$ are erected externally on the sides of any triangle $A B C$, their circumcentres form a triangle similar to the three triangles.

I.M. Yaglom proves in $[8, \mathrm{I} .2,22]$ the following generalisation of the theorem above:

On the sides of an arbitrary triangle $A B C$, exterior to it, isosceles triangles $C B A_{1}, A C B_{1}, B A C_{1}$ are erected with angles at the vertices $A_{1}, B_{1}$, and $C_{1}$, respectively equal to $\alpha, \beta$, and $\gamma$. If $\alpha+\beta+\gamma=2 \pi$, then the angles of the triangle $A_{1} B_{1} C_{1}$ are equal to $\frac{1}{2} \alpha, \frac{1}{2} \beta$, and $\frac{1}{2} \gamma$.

Note that the case $\alpha=\beta=\gamma=\frac{2}{3} \pi$ is just the same as taking the centres of equilateral triangles. It is easy to check that these two generalisations are equivalent by decomposing each external triangle into three isosceles triangles that have a common vertex in the circumcentre (note that the angles at the circumcentre are just the double of the angles of the triangle).

Considering the formulation of Yaglom, we drop the condition $\alpha+\beta+\gamma=2 \pi$ and repeat the transformation to obtain an infinite sequence of triangles. In doing so, the angles $\alpha$, $\beta$, and $\gamma$ stay fixed. We analyse two cases. In the first case, all three angles are the same. In the second case, two angles coincide and the third equals $\pi$ (hence, the corresponding erected triangle is degenerate). Equivalently to the second case, we may erect only two similar isosceles triangles and take the centre of the remaining side as the third vertex of the new triangle. We prove that in both cases, the shape of the triangles converge to the shape of the triangle one would get if the condition $\alpha+\beta+\gamma=2 \pi$ were satisfied. That is an equilateral triangle in the first case and a rectangular isosceles triangle in the second case.

In this article $\Delta_{0}$ always denotes the initial triangle with vertices $A_{0}, B_{0}$, and $C_{0}$ (ordered counterclockwise). For $n \in \mathbb{N}$ the points $A_{n+1}, B_{n+1}$, and $C_{n+1}$ are defined recursively such that $A_{n} C_{n} B_{n+1}, B_{n} A_{n} C_{n+1}, C_{n} B_{n} A_{n+1}$ are isosceles triangles. The triangle with vertices $A_{n}, B_{n}$, and $C_{n}$ is denoted by $\Delta_{n}$. The side-lengths of $\Delta_{n}$ are denoted by $x_{n}:=$ $\overline{B_{n} C_{n}}, y_{n}:=\overline{C_{n} A_{n}}$, and $z_{n}:=\overline{A_{n} B_{n}}$ and the angles are denoted by $\alpha_{n}:=\angle B_{n} A_{n} C_{n}$, $\beta_{n}:=\angle C_{n} B_{n} A_{n}$, and $\gamma_{n}:=\angle A_{n} C_{n} B_{n}$.

We do not demand the triangle to be non-degenerate. The degenerate case where all three points are pairwise distinct but on a common line will be used to show that some given bounds are sharp. In the other degenerate cases there have to be vertices of the triangle that coincide and hence there has to be a side of length 0 . Although such a side has no direction, this does not cause any problems for the iteration since in this case, the erected triangle always degenerates to a single point. Thus, the new vertex coincides with the two old ones. As a direct consequence, the degenerate case where all three points coincide is without any interest since such a triangle is invariant under all transformations introduced above. Therefore, we exclude the case $A_{0}=B_{0}=C_{0}$ for all of this article.

\section{Equilateral case}

In this section, the triangles erected externally on the sides of $\Delta_{n}$ are similar to each other. More precisely, there is an angle $0<\theta<\pi / 2$ such that $\angle A_{n+1} B_{n} C_{n}=\angle B_{n} C_{n} A_{n+1}=$ 


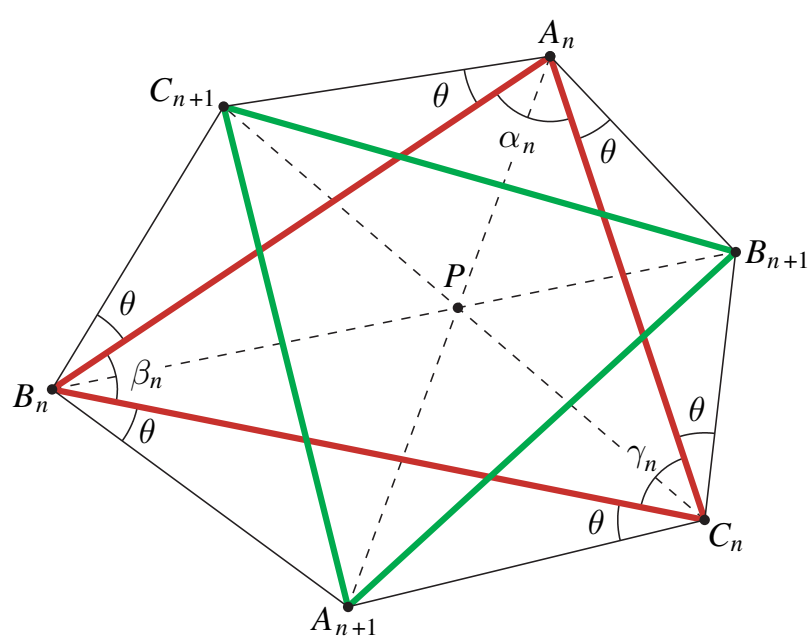

Fig. 1 Transformation with similar isosceles externally erected triangles

$\angle B_{n+1} C_{n} A_{n}=\angle C_{n} A_{n} B_{n+1}=\angle C_{n+1} A_{n} B_{n}=\angle A_{n} B_{n} C_{n+1}=\theta$. The so erected triangles are also known as Kiepert triangles, see [2]. The main result of this section can also be found in [5] where the convergence of this transformation is studied by considering the dominating eigenvalue. In this paper, we give a different proof of this result, which allows us a more detailed view at the process of convergence.

Remark 1.1. The lines $A_{n} A_{n+1}, B_{n} B_{n+1}$, and $C_{n} C_{n+1}$ meet in a common point $P$, see [2] for a proof. Note that this point is inside the triangle $\Delta_{n}$ if and only if all angles of $\Delta_{n}$ are $<\pi-\theta$.

We first show how the side-lengths of the triangle $\Delta_{n+1}$ can be expressed in terms of the preceding triangle. Regarding the transformation there is no distinction between $x_{n}, y_{n}$, and $z_{n}$. Therefore we state the claims usually for only one instance, but we will use in the following the analogue statements as well.

Lemma 1.2. Let $E_{n}$ denote the area of $\Delta_{n}$. Then the following identities hold:

$$
\begin{aligned}
x_{n+1}^{2} & =\frac{1}{2} \tan ^{2} \theta \cdot\left(y_{n}^{2}+z_{n}^{2}\right)+\frac{1}{4}\left(1-\tan ^{2} \theta\right) \cdot x_{n}^{2}+2 \tan \theta \cdot E_{n}, \\
x_{n+1}^{2}-y_{n+1}^{2} & =\frac{1}{4}\left(1-3 \tan ^{2} \theta\right)\left(x_{n}^{2}-y_{n}^{2}\right) .
\end{aligned}
$$

Proof. Note that $\overline{A_{n} C_{n+1}}=1 /(2 \cos \theta) \cdot z_{n}$ and $\overline{A_{n} B_{n+1}}=1 /(2 \cos \theta) \cdot y_{n}$. Hence, applying the law of cosines to the triangle $A_{n} C_{n+1} B_{n+1}$ yields

$$
\begin{aligned}
x_{n+1}^{2} & =\frac{1}{4 \cos ^{2} \theta} \cdot y_{n}^{2}+\frac{1}{4 \cos ^{2} \theta} \cdot z_{n}^{2}-2 \cdot \frac{1}{4 \cos ^{2} \theta} \cdot y_{n} z_{n} \cos \left(2 \theta+\alpha_{n}\right) \\
& =\frac{1}{4 \cos ^{2} \theta}\left(y_{n}^{2}+z_{n}^{2}\right)-\frac{\cos \left(2 \theta+\alpha_{n}\right)}{2 \cos ^{2} \theta} \cdot y_{n} z_{n} .
\end{aligned}
$$


Using the addition theorems for the cosine and the sine we obtain $\cos \left(2 \theta+\alpha_{n}\right)=\left(\cos ^{2} \theta-\right.$ $\left.\sin ^{2} \theta\right) \cos \alpha_{n}-2 \sin \theta \cos \theta \sin \alpha_{n}$. Applying this and $E_{n}=\left(\sin \alpha_{n} / 2\right) y_{n} z_{n}$ to the identity above leads to

$$
\begin{aligned}
x_{n+1}^{2} & =\frac{1}{4 \cos ^{2} \theta}\left(y_{n}^{2}+z_{n}^{2}\right)-\frac{\left(1-\tan ^{2} \theta\right)}{2} \cos \alpha_{n} \cdot y_{n} z_{n}+\tan \theta \sin \alpha_{n} \cdot y_{n} z_{n} \\
& =\frac{1}{4 \cos ^{2} \theta}\left(y_{n}^{2}+z_{n}^{2}\right)-\frac{1-\tan ^{2} \theta}{2} \cdot \frac{y_{n}^{2}+z_{n}^{2}-x_{n}^{2}}{2}+2 \tan \theta \cdot E_{n} \\
& =\left(\frac{1-\cos ^{2} \theta}{4 \cos ^{2} \theta}+\frac{1}{4} \tan ^{2} \theta\right) \cdot\left(y_{n}^{2}+z_{n}^{2}\right)+\frac{1}{4}\left(1-\tan ^{2} \theta\right) \cdot x_{n}^{2}+2 \tan \theta \cdot E_{n} \\
& =\frac{1}{2} \tan ^{2} \theta \cdot\left(y_{n}^{2}+z_{n}^{2}\right)+\frac{1}{4}\left(1-\tan ^{2} \theta\right) \cdot x_{n}^{2}+2 \tan \theta \cdot E_{n} .
\end{aligned}
$$

The second equation is a direct consequence of the first one together with its analogue for $y_{n+1}$.

Corollary 1.3. Let $x_{n} \geq y_{n}$. Then

$$
\begin{array}{ll}
x_{n+1} \geq y_{n+1} & \text { if } \quad \theta \leq \frac{\pi}{6} \quad \text { and } \\
x_{n+1} \leq y_{n+1} & \text { if } \quad \theta \geq \frac{\pi}{6},
\end{array}
$$

where equality on the left-hand sides holds if and only if $\theta=\pi / 6$ or $x_{n}=y_{n}$.

Proof. This is a direct consequence of (1.2) since $\tan ^{2}(\pi / 6)=1 / 3$.

Remark 1.4. We may assume that in the initial triangle $x_{0}$ is the greatest side and $z_{0}$ is the smallest. If $\theta<\pi / 6$, the corollary above implies that $x_{n}$ is the greatest side of $\Delta_{n}$ and $z_{n}$ is the smallest one for every $n$. If $\theta>\pi / 6$, things are different. For even $n$, we still have $x_{n} \geq y_{n} \geq z_{n}$, whereas $x_{n} \leq y_{n} \leq z_{n}$ holds for odd $n$. In the special case $\theta=\pi / 6$, the triangle $\Delta_{n}$ is equilateral for every $n>0$. This observation is a motivation to consider the two subsequences $\left(\Delta_{2 n}\right)_{n \in \mathbb{N}}$ and $\left(\Delta_{2 n+1}\right)_{n \in \mathbb{N}}$ sometimes separately.

To study the behaviour of corresponding side-lengths during the iteration, we state some estimates. First, note the following two simple inequalities, which will be used several times.

Lemma 1.5. Let $r$ and $s$ be two positive real numbers. Then $r^{2}+s^{2} \geq \frac{1}{2}(r+s)^{2}$ and $r^{2}+s^{2} \geq 2 r s$.

Proof. Since $(r-s)^{2}$ is positive, we obtain $4 r^{2}+4 s^{2} \geq\left(4 r^{2}-(r-s)^{2}\right)+\left(4 s^{2}-(s-r)^{2}\right)=$ $(3 r-s)(r+s)+(3 s-r)(s+r)=(2 r+2 s)(r+s)$. Subtracting $2\left(r^{2}+s^{2}\right)$ on both sides implies the second claim. 
Lemma 1.6. For the corresponding side-lengths of subsequent triangles, the following lower bounds hold:

$$
\begin{aligned}
& x_{n+1} \geq \frac{1}{2} x_{n}, \\
& x_{n+2}^{2} \geq \frac{1}{16}\left(9 \tan ^{4} \theta+1\right) \cdot x_{n}^{2} .
\end{aligned}
$$

Moreover, if $\Delta_{n}$ is non-degenerate, both bounds are strict.

Proof. By Lemma 1.5 we obtain $y_{n}^{2}+z_{n}^{2} \geq \frac{1}{2}\left(y_{n}+z_{n}\right)^{2} \geq \frac{1}{2} x_{n}^{2}$. Applying this to (1.1) yields

$$
x_{n+1}^{2} \geq \frac{1}{4} \tan ^{2} \theta \cdot x_{n}^{2}+\frac{1}{4}\left(1-\tan ^{2} \theta\right) \cdot x_{n}^{2}+2 \tan \theta \cdot E_{n} \geq \frac{1}{4} x_{n}^{2} .
$$

Now the first inequality follows directly. In the last step we subtracted $2 \tan \theta \cdot E_{n}$. Since $\tan \theta>0$, equality cannot occur if $\Delta_{n}$ is non-degenerate. Using the analogues of (1.1) provides the following identity:

$$
\begin{aligned}
y_{n+1}^{2}+z_{n+1}^{2} & =\frac{\tan ^{2} \theta}{2}\left(2 x_{n}^{2}+y_{n}^{2}+z_{n}^{2}\right)+\frac{1-\tan ^{2} \theta}{4}\left(y_{n}^{2}+z_{n}^{2}\right)+4 \tan \theta \cdot E_{n} \\
& =\tan ^{2} \theta \cdot x_{n}^{2}+\frac{1+\tan ^{2} \theta}{4}\left(y_{n}^{2}+z_{n}^{2}\right)+4 \tan \theta \cdot E_{n} .
\end{aligned}
$$

We apply this to the analogue of (1.1) for $x_{n+2}^{2}$ :

$$
\begin{aligned}
x_{n+2}^{2} \geq & \frac{1}{2} \tan ^{2} \theta\left(\tan ^{2} \theta \cdot x_{n}^{2}+\frac{1+\tan ^{2} \theta}{4}\left(y_{n}^{2}+z_{n}^{2}\right)+4 \tan \theta \cdot E_{n}\right) \\
& \quad+\frac{1-\tan ^{2} \theta}{4}\left(\frac{\tan ^{2} \theta}{2}\left(y_{n}^{2}+z_{n}^{2}\right)+\frac{1-\tan ^{2} \theta}{4} \cdot x_{n}^{2}+2 \tan \theta \cdot E_{n}\right) \\
= & \frac{\tan ^{2} \theta}{4}\left(y_{n}^{2}+z_{n}^{2}\right)+\frac{8 \tan ^{4} \theta+\left(1-\tan ^{2} \theta\right)^{2}}{16} \cdot x_{n}^{2}+\frac{3 \tan ^{3} \theta+\tan \theta}{2} \cdot E_{n} \\
\geq & \frac{\tan ^{2} \theta}{8} \cdot x_{n}^{2}+\frac{9 \tan ^{4} \theta-2 \tan ^{2} \theta+1}{16} \cdot x_{n}^{2} \\
= & \frac{9}{16} \tan ^{4} \theta \cdot x_{n}^{2}+\frac{1}{16} x_{n}^{2} .
\end{aligned}
$$

In the last but one step we subtracted $\left(3 \tan ^{3} \theta+\tan \theta\right) E_{n} / 2$. Since $3 \tan ^{3} \theta+\tan \theta>0$, equality cannot occur if $\Delta_{n}$ is non-degenerate.

The first lower bound given in the previous lemma is sharp as one can check by considering the degenerate case $\alpha_{n}=\pi$ and $y_{n}=z_{n}$. The second lower bound is sharp, too. This can be seen by considering again the degenerate case $\alpha_{n}=\pi$ and $y_{n}=z_{n}$ while $\theta$ tends to 0 . As a consequence of these two estimates, we state the following theorems.

Theorem 1.7. For $n \geq 1$, the side-lengths of $\Delta_{n}$ are all $>0$. 
Proof. We may assume that $\Delta_{n-1}$ is degenerate since otherwise the claim follows directly from (1.3).

Since $\Delta_{0}$ has at least one side of length $>0,(1.3)$ implies that every triangle has at least one side of length $>0$. Suppose $x_{n}=0$. Then (1.3) yields $x_{n-1}=0$. Thus, $E_{n-1}=0$ and consequently, $x_{n}^{2}=\tan ^{2} \theta \cdot\left(y_{n-1}^{2}+z_{n-1}^{2}\right) / 2$ by (1.1). We know $y_{n-1}^{2}+z_{n-1}^{2}>0$ since otherwise all vertices of $\Delta_{n-1}$ would coincide. With $\tan ^{2} \theta>0$ we obtain $x_{n}>0$, a contradiction.

Theorem 1.8. We have:

$$
\begin{aligned}
& \text { If } 0<\theta<\frac{\pi}{6}, \text { then } \quad \lim _{n \rightarrow \infty} x_{n}=0 . \\
& \text { If } \frac{\pi}{6}<\theta<\frac{\pi}{2}, \text { then } \quad \lim _{n \rightarrow \infty} x_{n}=\infty . \\
& \text { If } \theta=\frac{\pi}{6}, \text { then } x_{n}=x_{1} \quad \forall n>0 .
\end{aligned}
$$

Proof. First let $0<\theta<\pi / 6$. Assume $x_{0} \geq y_{0} \geq z_{0}$. Then $x_{n} \geq y_{n} \geq z_{n}$ for every $n$ by Remark 1.4. Hence, $\lim _{n \rightarrow \infty} x_{n}=0$ implies $\lim _{n \rightarrow \infty} y_{n}=\lim _{n \rightarrow \infty} z_{n}=0$. Thus, it suffices to prove the claim for the case $x_{0} \geq y_{0} \geq z_{0}$. Since $x_{n} \geq y_{n} \geq z_{n}$, we obtain $\alpha_{n} \geq \beta_{n} \geq \gamma_{n}$ by the law of sines. This implies $\gamma_{n} \leq \pi / 6$ and hence, $\sin \gamma_{n} \leq \sqrt{3} / 2$. With $E_{n}=\left(\sin \gamma_{n} / 2\right) x_{n} y_{n}$, we obtain by (1.1)

$$
\begin{aligned}
x_{n+1}^{2} & \leq \frac{1}{2} \tan ^{2} \theta \cdot\left(y_{n}^{2}+z_{n}^{2}\right)+\frac{1}{4}\left(1-\tan ^{2} \theta\right) \cdot x_{n}^{2}+\frac{\sqrt{3}}{2} \tan \theta \cdot x_{n} y_{n} \\
& \leq\left(\frac{1}{4}+\frac{3}{4} \tan ^{2} \theta+\frac{\sqrt{3}}{2} \tan \theta\right) \cdot x_{n}^{2} .
\end{aligned}
$$

Now $\theta<\pi / 6$ implies $\tan \theta<\sqrt{3} / 3$ and consequently $\frac{1}{4}+\frac{3}{4} \tan ^{2} \theta+\frac{\sqrt{3}}{2} \tan \theta<1$. The claim follows.

Now let $\pi / 6<\theta<\pi / 2$. Assume $x_{0} \leq y_{0} \leq z_{0}$. Then $x_{2 n} \leq y_{2 n} \leq z_{2 n}$ for every $n$ by Remark 1.4. Hence, $\lim _{n \rightarrow \infty} x_{2 n}=\infty$ implies $\lim _{n \rightarrow \infty} y_{2 n}=\lim _{n \rightarrow \infty} z_{2 n}=\infty$. Thus, to prove $\lim _{n \rightarrow \infty} x_{2 n}=\infty$ it suffices to consider the case $x_{0} \leq y_{0} \leq z_{0}$. Let $n$ be even. Since $x_{n} \leq y_{n} \leq z_{n}$, we obtain $\alpha_{n} \leq \beta_{n} \leq \gamma_{n}$. This implies $\gamma_{n} \geq \pi / 6$ and hence, $\sin \gamma_{n} \geq \sqrt{3} / 2$. With $E_{n}=\left(\sin \gamma_{n} / 2\right) x_{n} y_{n}$, we obtain by (1.1)

$$
\begin{aligned}
z_{n+1}^{2} & \geq \frac{1}{2} \tan ^{2} \theta \cdot\left(x_{n}^{2}+y_{n}^{2}\right)+\frac{1}{4}\left(1-\tan ^{2} \theta\right) \cdot z_{n}^{2}+\frac{\sqrt{3}}{2} \tan \theta \cdot x_{n} y_{n} \\
& \geq\left(\frac{1}{4}+\frac{3}{4} \tan ^{2} \theta+\frac{\sqrt{3}}{2} \tan \theta\right) \cdot x_{n}^{2} .
\end{aligned}
$$

Now $\theta<\pi / 6$ implies $\tan \theta<\sqrt{3} / 3$ and consequently $\kappa:=\frac{1}{4}+\frac{3}{4} \tan ^{2} \theta+\frac{\sqrt{3}}{2} \tan \theta>1$. Since $z_{n+1} \leq y_{n+1} \leq x_{n+1}$ by Remark 1.4, we conclude analogously $x_{n+2}^{2} \geq \kappa z_{n+1}^{2}$. Thus, $x_{n+2}^{2} \geq \kappa^{2} x_{n}^{2}$ whenever $n$ is even and consequently $\lim _{n \rightarrow \infty} x_{2 n}=\infty$. By analogous reasons we obtain $\lim _{n \rightarrow \infty} x_{2 n+1}=\infty$ and the claim follows. 
In the last case, we conclude by Corollary 1.3 that for every $n>0$ the triangle $\Delta_{n}$ is equilateral. Furthermore, $\tan \theta=\sqrt{3} / 3$. Thus, for $n>0,(1.1)$ yields $x_{n+1}^{2}=x_{n}^{2}$.

Due to the unbounded growth of the triangles for $\theta>\pi / 6$ and the fact that for $\theta<\pi / 6$, the triangle sequence collapses to a single point, the only reasonable case to study seems to be the case where $\theta$ equals $\pi / 6$. However, since we are interested in the shape of the triangle, the size of the triangle does not matter.

We state another two simple inequalities for our further estimates.

Lemma 1.9. Let $r, s$, and $t$ be positive real numbers all smaller than 1 . Then $1-s^{2}<$ $r\left(1-t^{2}\right)$ implies $(1-s)<r(1-t)$.

Proof. We obtain $1-s^{2}<1-t^{2}$ and therefore $s>t$. Thus, $(1-s)=\left(1-s^{2}\right) /(1+s)<$ $r\left(1-t^{2}\right) /(1+s)<r\left(1-t^{2}\right) /(1+t)<r(1-t)$.

The following general proposition applies separately to the two triangle sequences, i.e. the one with the even and the one with the odd indices. As in Remark 1.4 we assume $x_{0} \geq y_{0}$ for the following proposition.

Proposition 1.10. Let $x_{0}>y_{0}$. Then for every angle $\theta$, there is a constant $0 \leq \kappa<1$ such that

$$
0 \leq\left|1-\frac{y_{n+2}}{x_{n+2}}\right| \leq \kappa\left|1-\frac{y_{n}}{x_{n}}\right|,
$$

where equality holds if and only if $\theta=\pi / 6$ or $y_{n}=x_{n}$.

Proof. First note that for $n \geq 1$, Theorem 1.7 states $x_{n}>0$. Furthermore, since $x_{0} \geq y_{0}$ and the case $A_{0}=B_{0}=C_{0}$ is excluded, we obtain $x_{0}>0$. Now, (1.2) provides

$$
x_{n+2}^{2}-y_{n+2}^{2}=\left(\frac{1}{4}-\frac{3}{4} \tan ^{2} \theta\right)^{2} \cdot\left(x_{n}^{2}-y_{n}^{2}\right)
$$

and hence,

$$
1-\frac{y_{n+2}^{2}}{x_{n+2}^{2}}=\frac{\left(1-3 \tan ^{2} \theta\right)^{2}}{16} \cdot \frac{x_{n}^{2}}{x_{n+2}^{2}}\left(1-\frac{y_{n}^{2}}{x_{n}^{2}}\right) .
$$

Thus, we may assume $x_{n} \neq y_{n}$ since otherwise we are done. Applying (1.4) yields

$$
\left|1-\frac{y_{n+2}^{2}}{x_{n+2}^{2}}\right| \leq \frac{\left(1-3 \tan ^{2} \theta\right)^{2}}{1+9 \tan ^{4} \theta}\left|1-\frac{y_{n}^{2}}{x_{n}^{2}}\right| .
$$

Since $0 \leq\left(1-3 \tan ^{2} \theta\right)^{2}<1+9 \tan ^{4} \theta$ the claim follows for $\kappa:=\left(1-3 \tan ^{2} \theta\right)^{2} /(1+$ $9 \tan ^{4} \theta$ ) by Lemma 1.9. Note that $\kappa=0$ if and only if $\theta=\pi / 6$.

For $\theta \leq \pi / 4$, the ratio of side-lengths tends to 1 in every step of the iteration. In other terms, the function $n \mapsto 1-\min \left\{x_{n}, y_{n}\right\} / \max \left\{x_{n}, y_{n}\right\}$ is strictly decreasing as long as the values differ from 0 . Note that for $\theta>\pi / 6$, the role of the smaller side-length alternates. 
Proposition 1.11. Let $x_{0}>y_{0}$ and $\theta \neq \pi / 6$. Then there is a positive constant $\kappa<1$ that only depends on $\theta$ such that the following holds:

$$
\begin{array}{ll}
0<1-\frac{y_{n+1}}{x_{n+1}} \leq \kappa\left(1-\frac{y_{n}}{x_{n}}\right) & \text { if } \quad \theta<\frac{\pi}{6} \\
0<1-\frac{x_{n+1}}{y_{n+1}} \leq \kappa\left(1-\frac{y_{n}}{x_{n}}\right) & \text { if } \quad \frac{\pi}{6}<\theta \leq \frac{\pi}{4} \text { and } n \text { even, } \\
0<1-\frac{y_{n+1}}{x_{n+1}} \leq \kappa\left(1-\frac{x_{n}}{y_{n}}\right) & \text { if } \quad \frac{\pi}{6}<\theta \leq \frac{\pi}{4} \text { and } n \text { odd } .
\end{array}
$$

Proof. First note that $x_{n}>0$ and $y_{n}>0$ for $n \geq 1$ by Theorem 1.7. Moreover, $x_{0}>0$ since $x_{0} \geq y_{0}$ and the case $A_{0}=B_{0}=C_{0}$ is excluded.

We set $\omega:=1-3 \tan ^{2} \theta$. First assume $\theta<\pi / 6$. Then $x_{n}>y_{n}$ by Corollary 1.3. Furthermore, $0<\tan ^{2} \theta<1 / 3$ and therefore $0<\omega<1$. By (1.3) we know $x_{n} \leq 2 x_{n+1}$. Hence, dividing both sides of (1.2) by $x_{n+1}^{2}$ provides

$$
1-\frac{y_{n+1}^{2}}{x_{n+1}^{2}}=\frac{\omega}{4} \cdot \frac{x_{n}^{2}}{x_{n+1}^{2}}\left(1-\frac{y_{n}^{2}}{x_{n}^{2}}\right) \leq \omega\left(1-\frac{y_{n}^{2}}{x_{n}^{2}}\right) .
$$

Now the claim follows from Lemma 1.9 by setting $\kappa:=\omega$.

For $\theta>\pi / 6$, we know by Corollary 1.3 that $x_{n}>y_{n}$ if $n$ is even and $y_{n}>x_{n}$ otherwise. We restrict ourselves to the case where $n$ is even. The other case can be obtained by exchanging $x_{n}$ with $y_{n}, x_{n+1}$ with $y_{n+1}$, and $z_{0}$ with $z_{1}$. Note that $\omega<0$ for $\theta>\pi / 6$.

We assume $\pi / 6<\theta<\pi / 4$. Then $\tan ^{2} \theta \leq 1$. Hence, (1.1) yields $y_{n+1}^{2} \geq \frac{1}{2}\left(x_{n}^{2}+\right.$ $\left.z_{n}^{2}\right) \tan ^{2} \theta$. Furthermore, $\tan ^{2} \theta \leq 1$ implies $-\omega \leq 2 \tan ^{2} \theta$. We divide both sides of (1.2) by $-y_{n+1}^{2}$ and obtain

$$
\begin{aligned}
1-\frac{x_{n+1}^{2}}{y_{n+1}^{2}} & =\frac{-\omega}{4} \cdot \frac{x_{n}^{2}}{y_{n+1}^{2}}\left(1-\frac{y_{n}^{2}}{x_{n}^{2}}\right) \\
& \leq \frac{\tan ^{2} \theta}{2} \cdot \frac{2 x_{n}^{2}}{\left(x_{n}^{2}+z_{n}^{2}\right) \tan ^{2} \theta}\left(1-\frac{y_{n}^{2}}{x_{n}^{2}}\right) \\
& =\frac{x_{n}^{2}}{x_{n}^{2}+z_{n}^{2}}\left(1-\frac{y_{n}^{2}}{x_{n}^{2}}\right) .
\end{aligned}
$$

Now set $\varepsilon:=\min \left\{1,\left(z_{0} / x_{0}\right)^{2}\right\}$. Then Proposition 1.10 together with induction implies $z_{n}^{2} \geq \varepsilon x_{n}^{2}$. The claim follows for $\kappa:=1 /(1+\varepsilon)$ by using Lemma 1.9.

Remark 1.12. For $\theta>\pi / 4$ and $x_{n}>y_{n}$, it is possible that $1-x_{n+1} / y_{n+1}$ exceeds $1-y_{n} / x_{n}$, especially if $\theta$ is close to $\pi / 2$. However, in this situation there is another observation one can make: While $\theta$ tends to $\pi / 2$, the angle $\alpha_{2 n}$ tends to $\alpha_{0}$ for every $n \in \mathbb{N}$. Analogously, $\lim _{\theta \rightarrow \pi / 2} \beta_{2 n}=\beta_{0}$ and $\lim _{\theta \rightarrow \pi / 2} \gamma_{2 n}=\gamma_{0}$. Hence, the shape of $\Delta_{2}$ tends to the shape of $\Delta_{0}$. On the other hand $\lim _{\theta \rightarrow \pi / 2} x_{1}=\lim _{\theta \rightarrow \pi / 2} y_{1}=\lim _{\theta \rightarrow \pi / 2} z_{1}=\infty$ as long as $\Delta_{0}$ is non-degenerate. Thus, one cannot speak of a limit triangle. 
To avoid the enormous growth of the triangles, one can dilate each transformed triangle after the iteration with the reciprocal of the largest side-length. Equivalently, one can apply this dilation before the step of iteration. By doing so, $\Delta_{1}$ converges pointwise while $\theta$ tends to $\pi / 2$ as long as we take a fixed centre for the dilations. Thus, we obtain a limit triangle which we call $\Delta_{1}^{\prime}$. Repeating this process leads to two sequences of pairwise similar triangles $\Delta_{2 n}^{\prime}$ and $\Delta_{2 n+1}^{\prime}$. The triangles $\Delta_{0}$ and $\Delta_{1}^{\prime}$ do not have to be similiar.

Clearly, while $\theta$ tends to $\pi / 2$, the factor of the dilation we apply to $\Delta_{0}$ tends to 0 . Thus, the vertices $A_{1}^{\prime}, B_{1}^{\prime}$, and $C_{1}^{\prime}$ of $\Delta_{1}^{\prime}$ lie on the lines through the dilation centre that are perpendicular to one of the sides of $\Delta_{0}$. Moreover, the proportions of the distances from the dilation centre to $A_{1}^{\prime}, B_{1}^{\prime}, C_{1}^{\prime}$ match the proportions of $z_{1}, y_{1}, x_{1}$. Hence, a triangle similar to $\Delta_{1}^{\prime}$ can be obtained by taking three concurrent rays $r_{0}, r_{1}, r_{2}$ such that $r_{0}$ and $r_{1}$ span the angle $2 \alpha_{0}, r_{0}$ and $r_{2}$ span the angle $2 \beta_{0}$, and $r_{1}$ and $r_{2}$ span the angle $2 \gamma_{0}$. Taking the points on $r_{0}, r_{1}, r_{2}$ at distance $z_{0}, y_{0}, x_{0}$, respectively, to the intersection of the three rays provides a triangle similar to $\Delta_{1}^{\prime}$.

Since $\Delta_{2}^{\prime}$ is similar to $\Delta_{0}$ again, the shape of $\Delta_{1}^{\prime}$ can be seen as some kind of dual shape to the shape of $\Delta_{0}$.

The following theorem is our main result, namely, regarding only the shape of the triangles, $\Delta_{n}$ tends to an equilateral triangle for $n \rightarrow \infty$.

Theorem 1.13. For every initial triangle $\Delta_{0}$ and every angle $0<\theta<\pi / 2$, the following two limits hold:

$$
\lim _{n \rightarrow \infty} \frac{x_{n}}{y_{n}}=1, \quad \lim _{n \rightarrow \infty} \alpha_{n}=\frac{\pi}{3} .
$$

Proof. The first limit is a direct consequence of Proposition 1.10. The second limit follows by the first together with the law of sines.

We conclude this section by stating two theorems concerning the position and the orientation of the triangles.

Theorem 1.14. For every $n>0$, the centroid of $\Delta_{n}$ coincides with the centroid of $\Delta_{0}$.

Proof. We consider the Euclidean plane as vector space. For $n \in \mathbb{N}$, let $a_{n}, b_{n}$, and $c_{n}$ be the vectors representing the points $A_{n}, B_{n}$, and $C_{n}$, respectively. Let $\delta$ be the linear transformation that rotates the Euclidean plane by $\pi / 2$. Then $a_{n+1}=\frac{1}{2}\left(b_{n}+c_{n}\right)+\left(\frac{1}{2} \tan \theta\left(b_{n}-\right.\right.$ $\left.\left.c_{n}\right)\right)^{\delta}$. Since $\delta$ is linear, this implies $a_{n+1}+b_{n+1}+c_{n+1}=a_{n}+b_{n}+c_{n}$. Thus, the centroid of $\Delta_{n}$, defined as $\frac{1}{3}\left(a_{n}+b_{n}+c_{n}\right)$ coincides with the one of $\Delta_{n+1}$. The claim follows by induction.

Theorem 1.15. For every $n>0$, the triangle $\Delta_{n}$ is non-degenerate and counterclockwise oriented.

Proof. Assume that $\Delta_{n}$ is non-degenerate and counterclockwise oriented. By symmetric reasons we may assume $x_{n} \geq y_{n} \geq z_{n}$. Hence, $\alpha_{n} \geq \beta_{n} \geq \gamma_{n}$ by the law of sines and therefore $\beta_{n} \leq \pi / 2$ and $\gamma_{n} \leq \pi / 2$. 
Let $A_{n}^{\prime}, B_{n}^{\prime}$, and $C_{n}^{\prime}$ denote the centres of $B_{n} C_{n}, C_{n} A_{n}$, and $A_{n} B_{n}$, respectively. Since the triangle $A_{n}^{\prime} C_{n} B_{n}^{\prime}$ is similar to $\Delta_{n}$, we obtain $\angle C_{n} A_{n}^{\prime} B_{n}^{\prime}=\beta_{n}$. On the other hand, let $l$ be the perpendicular bisector of the side $C_{n} A_{n}$, i.e. the line through $B_{n}^{\prime}$ and $B_{n+1}$. Since $x_{n} \geq z_{n}$, the line $l$ intersects $x_{n}$ in a point $S$. We obtain $\angle C_{n} S B_{n+1}=\pi / 2-\gamma_{n}$. Thus, $\min \left\{\beta_{n}, \pi / 2-\gamma_{n}\right\} \leq \angle C_{n} A_{n}^{\prime} B_{n+1} \leq \max \left\{\beta_{n}, \pi / 2-\gamma_{n}\right\}$ and therefore $0<$ $\angle C_{n} A_{n}^{\prime} B_{n+1}<\pi / 2$. Analogously, $0<\angle C_{n+1} A_{n}^{\prime} B_{n}<\pi / 2$. This implies that the angles $\angle C_{n+1} A_{n}^{\prime} A_{n+1}$ and $\angle A_{n+1} A_{n}^{\prime} B_{n+1}$ are greater than $\pi / 2$ and smaller than $\pi$ and consequently, $\angle B_{n+1} A_{n}^{\prime} C_{n+1}<\pi$. We conclude that $A_{n}^{\prime}$ is inside the triangle $\Delta_{n+1}$ and $\Delta_{n+1}$ is counterclockwise oriented since $\angle A_{n+1} A_{n}^{\prime} B_{n+1}<\pi$. Now the claim follows by induction.

\section{Rectangular isosceles case}

As in the previous section, the points $B_{n+1}$ and $C_{n+1}$ are the apices of similar isosceles triangles erected to the outside of $\Delta_{n}$ over the edges $y_{n}$ and $z_{n}$, respectively. More precisely, there is an angle $0<\theta<\pi / 2$ such that $\angle B_{n+1} C_{n} A_{n}=\angle C_{n} A_{n} B_{n+1}=\angle C_{n+1} A_{n} B_{n}=$ $\angle A_{n} B_{n} C_{n+1}=\theta$. In contrast to the previous section, the point $A_{n+1}$ is the centre of $B_{n} C_{n}$ (or, equivalently, the apex of a degenerate isosceles triangle with angle $\pi$ ).

Again, we first give equations for the side-lengths of the triangle $\Delta_{n+1}$ in terms of $\Delta_{n}$. Regarding the transformation there is no distinction between $y_{n}$ and $z_{n}$ except for the orientation. Therefore we state the claims usually for only one instance, but we will use the analogue statements as well in the following.

Lemma 2.1. Let $E_{n}$ be the area of $\Delta_{n}$. Then the following identities hold:

$$
\begin{aligned}
& x_{n+1}^{2}=\frac{1}{2} \tan ^{2} \theta \cdot\left(y_{n}^{2}+z_{n}^{2}\right)+\frac{1}{4}\left(1-\tan ^{2} \theta\right) \cdot x_{n}^{2}+2 \tan \theta \cdot E_{n}, \\
& y_{n+1}^{2}=\frac{1}{4} \cdot y_{n}^{2}+\frac{1}{4} \tan ^{2} \theta \cdot z_{n}^{2}+\tan \theta \cdot E_{n} .
\end{aligned}
$$

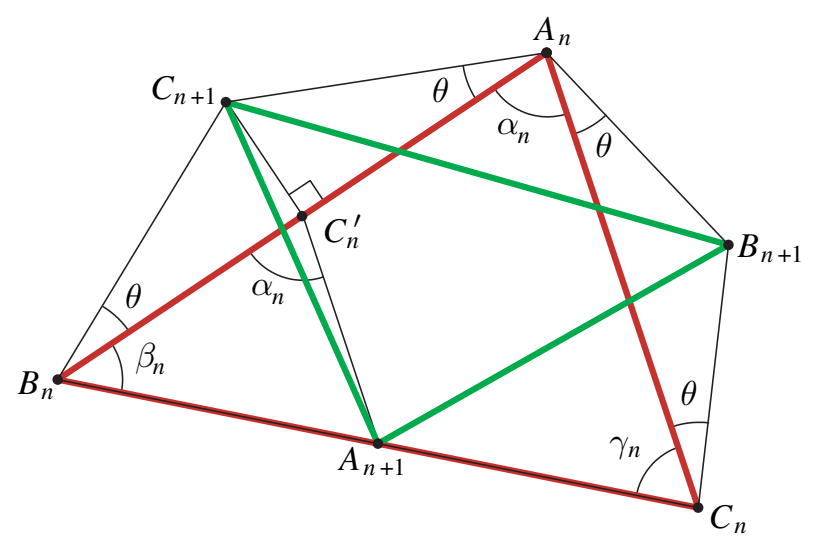

Fig. 2 Transformation with two similar isosceles externally erected triangles and one midpoint of a side 
Proof. The first equation is obtained in precisely the same way as in the proof of Lemma 1.2.

Let $C_{n}^{\prime}$ be the centre of $A_{n} B_{n}$. Then $\overline{C_{n+1} C_{n}^{\prime}}=\frac{1}{2} \tan \theta \cdot z_{n}$ and $\overline{A_{n+1} C_{n}^{\prime}}=\frac{1}{2} \cdot y_{n}$. Applying the law of cosines to the triangle $A_{n+1} C_{n}^{\prime} C_{n+1}$ (possibly degenerate for $\alpha_{n}=\pi / 2$ and oriented clockwise for $\alpha_{n}>\pi / 2$ ) yields

$$
y_{n+1}^{2}=\frac{1}{4} \cdot y_{n}^{2}+\frac{1}{4} \tan ^{2} \theta \cdot z_{n}^{2}-2 \cdot \frac{1}{4} \tan \theta \cdot y_{n} z_{n} \cos \left(\frac{\pi}{2}+\beta_{n}+\gamma_{n}\right) .
$$

With $\cos \left(\pi / 2+\beta_{n}+\gamma_{n}\right)=\sin \left(-\beta_{n}-\gamma_{n}\right)=\sin \left(\alpha_{n}-\pi / 2\right)=-\sin \left(\alpha_{n}\right)$ and $E_{n}=$ $\frac{1}{2} \sin \alpha_{n} \cdot y_{n} z_{n}$, the second identity follows.

The following identities are immediate consequences of the previous lemma.

$$
\begin{aligned}
y_{n+1}^{2}-z_{n+1}^{2} & =\frac{1}{4}\left(1-\tan ^{2} \theta\right) \cdot\left(y_{n}^{2}-z_{n}^{2}\right), \\
y_{n+1}^{2}+z_{n+1}^{2} & =\frac{1}{4}\left(1+\tan ^{2} \theta\right) \cdot\left(y_{n}^{2}+z_{n}^{2}\right)+2 \tan \theta \cdot E_{n}, \\
x_{n+1}^{2}-y_{n+1}^{2}-z_{n+1}^{2} & =\frac{1}{4}\left(1-\tan ^{2} \theta\right) \cdot\left(x_{n}^{2}-y_{n}^{2}+z_{n}^{2}\right) .
\end{aligned}
$$

Corollary 2.2. Let $y_{n} \geq z_{n}$. Then

$$
\begin{array}{ll}
y_{n+1} \geq z_{n+1} & \text { if } \quad \theta \leq \frac{\pi}{4} \quad \text { and } \\
y_{n+1} \leq z_{n+1} & \text { if } \quad \theta \geq \frac{\pi}{4},
\end{array}
$$

where equality on the left-hand side holds if and only if $\theta=\pi / 4$ or $y_{n}=z_{n}$.

Proof. This is a direct consequence of (2.2) since $\tan (\pi / 4)=1$.

Since $A_{n+1}$ is obtained in a different way than $B_{n+1}$ and $C_{n+1}$, there is no corresponding condition that involves $x_{n}$ and $x_{n+1}$. Our next step is to give lower bounds for the sidelengths after two steps of iteration.

Lemma 2.3. For the corresponding side-lengths of subsequent triangles, the following lower bounds hold:

$$
\begin{aligned}
& x_{n+2}^{2} \geq \frac{1}{16}\left(1+\tan ^{4} \theta\right) x_{n}^{2}, \\
& y_{n+2}^{2} \geq \frac{1}{16}\left(1+\tan ^{4} \theta\right) y_{n}^{2} .
\end{aligned}
$$


Proof. We apply (2.3) and (1.1) to the analogue of (1.1) for $x_{n+2}^{2}$ :

$$
\begin{aligned}
x_{n+2}^{2} \geq & \frac{\tan ^{2} \theta}{2}\left(\frac{1+\tan ^{2} \theta}{4} \cdot\left(y_{n}^{2}+z_{n}^{2}\right)+2 \tan \theta \cdot E_{n}\right) \\
& \quad+\frac{1-\tan ^{2} \theta}{4}\left(\frac{\tan ^{2} \theta}{2} \cdot\left(y_{n}^{2}+z_{n}^{2}\right)+\frac{1-\tan ^{2} \theta}{4} \cdot x_{n}^{2}+2 \tan \theta \cdot E_{n}\right) \\
= & \frac{\tan ^{2} \theta}{4} \cdot\left(y_{n}^{2}+z_{n}^{2}\right)+\frac{\left(1-\tan ^{2} \theta\right)^{2}}{16} \cdot x_{n}^{2}+\frac{\tan \theta+\tan ^{3} \theta}{2} \cdot E_{n} .
\end{aligned}
$$

Now Lemma 1.5 implies $y_{n}^{2}+z_{n}^{2} \geq\left(y_{n}+z_{n}\right)^{2} / 2 \geq x_{n}^{2} / 2$ and thus,

$$
\begin{aligned}
x_{n+2}^{2} & \geq \frac{\tan ^{2} \theta}{8} \cdot x_{n}^{2}+\frac{1-2 \tan ^{2} \theta+\tan ^{4} \theta}{16} \cdot x_{n}^{2} \\
& =\frac{1}{16} x_{n}^{2}+\frac{1}{16} \tan ^{4} \theta \cdot x_{n}^{2} .
\end{aligned}
$$

Using (2.1) repeatedly yields

$$
\begin{aligned}
y_{n+2}^{2} & \geq \frac{1}{4} y_{n+1}^{2}+\frac{1}{4} \tan ^{2} \theta \cdot z_{n+1}^{2} \\
& \geq \frac{1}{16} y_{n}^{2}+\frac{1}{8} \tan ^{2} \theta \cdot z_{n+1}^{2}+\frac{1}{16} \tan ^{4} \theta \cdot y_{n}^{2} \\
& \geq \frac{1}{16} y_{n}^{2}+\frac{1}{16} \tan ^{4} \theta \cdot y_{n}^{2} .
\end{aligned}
$$

As in the previous section, the lemma above motivates us to consider the sequence of triangles as two separated sequences.

Theorem 2.4. For $n \geq 1$, the side-lengths of $\Delta_{n}$ are all $>0$.

Proof. The side-length $x_{n}$ does not depend on $A_{n}$ and hence, for a given triangle $\Delta_{n-1}$ and a given angle $\theta$, the side-length $x_{n}$ is just the same as in the previous section. Thus, $x_{n}>0$ by Theorem 1.7 .

For $y_{n}$ and $z_{n}$, we may assume that $\Delta_{n-1}$ is degenerate since otherwise the claim follows directly from (2.1).

Since $\Delta_{0}$ has at least two sides of length $>0,(2.1)$ implies $y_{1}>0$ and analogously, $z_{1}>0$. Now the claim follows by induction using (2.1).

We proceed by studying the ratio of corresponding side-lengths.

Proposition 2.5. Let $y_{0} \geq z_{0}$. Then for every angle $0<\theta<\pi / 2$, there is a constant $0 \leq \kappa<1$ such that

$$
0 \leq\left|1-\frac{z_{n+2}}{y_{n+2}}\right| \leq \kappa\left|1-\frac{z_{n}}{y_{n}}\right|,
$$

where equality holds if and only $\theta=\pi / 4$ or $y_{n}=z_{n}$. 
Proof. Note that $y_{n}>0$ for $n \geq 1$ by Theorem 2.4. Moreover, $y_{0}>0$ since otherwise $z_{0}=y_{0}=0$ and hence, $A_{0}=B_{0}=C_{0}$. Now, (2.2) provides

$$
y_{n+2}^{2}-z_{n+2}^{2}=\left(\frac{1}{4}-\frac{1}{4} \tan ^{2} \theta\right)^{2} \cdot\left(y_{n}^{2}-z_{n}^{2}\right)
$$

and hence,

$$
1-\frac{z_{n+2}^{2}}{y_{n+2}^{2}}=\frac{\left(1-\tan ^{2} \theta\right)^{2}}{16} \cdot \frac{y_{n}^{2}}{y_{n+2}^{2}}\left(1-\frac{z_{n}^{2}}{y_{n}^{2}}\right) .
$$

We may assume $y_{n} \neq z_{n}$ since otherwise we are done. Applying (2.6) yields

$$
\left|1-\frac{z_{n+2}^{2}}{y_{n+2}^{2}}\right| \leq \frac{\left(1-\tan ^{2} \theta\right)^{2}}{1+\tan ^{4} \theta}\left|1-\frac{z_{n}^{2}}{y_{n}^{2}}\right| .
$$

Since $0 \leq\left(1-\tan ^{2} \theta\right)^{2}<1+\tan ^{4} \theta$ the claim follows for $\kappa:=\left(1-\tan ^{2} \theta\right)^{2} /\left(1+\tan ^{4} \theta\right)$ by Lemma 1.9 . Note that $\kappa=0$ if and only if $\theta=\pi / 4$.

Proposition 2.6. For every angle $\theta$, there is a constant $0 \leq \kappa<1$ such that

$$
0 \leq\left|1-\frac{y_{n+2}^{2}+z_{n+2}^{2}}{x_{n+2}^{2}}\right| \leq \kappa \cdot\left|1-\frac{y_{n}^{2}+z_{n}^{2}}{x_{n}^{2}}\right|,
$$

where equality holds if and only $\theta=\pi / 4$ or $x_{n}^{2}=y_{n}^{2}+z_{n}^{2}$.

Proof. By Theorem 2.4, the only possibility where one of the fractions is not defined is the case $n=0$ and $x_{0}=0$. In this case, the term on the right-hand side can be understood as a term of inifinite value, which makes the claim obviously true for this case. Formula (2.4) provides

$$
x_{n+2}^{2}-y_{n+2}^{2}-z_{n+2}^{2}=\left(\frac{1}{4}-\frac{1}{4} \tan ^{2} \theta\right)^{2} \cdot\left(x_{n}^{2}-y_{n}^{2}-z_{n}^{2}\right)
$$

and hence,

$$
1-\frac{y_{n+2}^{2}+z_{n+2}^{2}}{x_{n+2}^{2}}=\frac{\left(1-\tan ^{2} \theta\right)^{2}}{16} \cdot \frac{x_{n}^{2}}{x_{n+2}^{2}}\left(1-\frac{y_{n}^{2}+z_{n}^{2}}{x_{n}^{2}}\right) .
$$

We may assume $x_{n}^{2} \neq y_{n}^{2}+z_{n}^{2}$ since otherwise we are done. Applying (2.5) yields

$$
\left|1-\frac{y_{n+2}^{2}+z_{n+2}^{2}}{x_{n+2}^{2}}\right| \leq \frac{\left(1-\tan ^{2} \theta\right)^{2}}{1+\tan ^{4} \theta} \cdot\left|1-\frac{y_{n}^{2}+z_{n}^{2}}{x_{n}^{2}}\right| .
$$

For $\kappa:=\left(1-\tan ^{2} \theta\right)^{2} /\left(1+\tan ^{4} \theta\right)$, the claim follows since $0 \leq\left(1-\tan ^{2} \theta\right)^{2}<1+\tan ^{4} \theta$. Note that $\kappa=0$ if and only if $\theta=\pi / 4$.

We are now ready to state our main result. Regarding only the shape of the triangles, $\Delta_{n}$ tends to a rectangular isosceles triangle for $n \rightarrow \infty$. 
Theorem 2.7. For every initial triangle $\Delta_{0}$ and every angle $0<\theta<\pi / 2$, the following limits hold:

$$
\begin{array}{rlrl}
\lim _{n \rightarrow \infty} \frac{y_{n}^{2}+z_{n}^{2}}{x_{n}^{2}} & =1, & \lim _{n \rightarrow \infty} \alpha_{n}=\frac{\pi}{2}, \\
\lim _{n \rightarrow \infty} \frac{y_{n}}{z_{n}}=1, & \lim _{n \rightarrow \infty} \beta_{n}=\frac{\pi}{4} .
\end{array}
$$

Proof. The limits on the left-hand side are immediate consequences of Propositions 2.5 and 2.6. By the law of cosines we know $\cos \alpha_{n}=\frac{1}{2}\left(y_{n}^{2}+z_{n}^{2}-x_{n}^{2}\right) /\left(y_{n} z_{n}\right)$. Now the limits on the left-hand side imply $\lim _{n \rightarrow \infty} \cos \alpha_{n}=0$ and hence, $\lim _{n \rightarrow \infty} \alpha_{n}=\pi / 2$. The last limit follows from $\lim _{n \rightarrow \infty} y_{n} / z_{n}=1$ together with the law of sines.

As in the previous section, the size of the triangles becomes stable for only one specific choice of $\theta$. For every greater angle, the triangles grow unboundedly and for every smaller angle, the triangles collapse to a single point.

\section{Theorem 2.8.}

$$
\begin{array}{rlrlrl}
\text { If } & 0<\theta<\frac{\pi}{4}, & \text { then } & \lim _{n \rightarrow \infty} x_{n} & =\lim _{n \rightarrow \infty} y_{n}=0 . \\
\text { If } & \frac{\pi}{4}<\theta<\frac{\pi}{2}, \text { then } & \lim _{n \rightarrow \infty} x_{n}=\lim _{n \rightarrow \infty} y_{n}=\infty . & \\
\text { If } & \theta & =\frac{\pi}{4}, & \text { then } & x_{n}=x_{1}=\sqrt{2} \cdot y_{1}=\sqrt{2} \cdot y_{n} \quad \forall n>0 .
\end{array}
$$

Proof. First let $0<\theta<\pi / 4$. Then $\tan \theta<1$ and hence, (2.3) implies

$$
\begin{aligned}
y_{n+1}^{2}+z_{n+1}^{2} & <\frac{1}{2}\left(y_{n}^{2}+z_{n}^{2}\right)+\tan \theta \sin \alpha_{n} \cdot y_{n} z_{n} \\
& \leq \frac{1}{2}\left(y_{n}^{2}+z_{n}^{2}\right)+\tan \theta \cdot y_{n} z_{n} .
\end{aligned}
$$

With Lemma 1.5 we conclude $y_{n+1}^{2}+z_{n+1}^{2}<\frac{1}{2}(1+\tan \theta)\left(y_{n}^{2}+z_{n}^{2}\right)$. Since $\frac{1}{2}(1+\tan \theta)<1$, this implies $\lim _{n \rightarrow \infty}\left(y_{n}^{2}+z_{n}^{2}\right)=0$ and hence, $\lim _{n \rightarrow \infty} y_{n}=\lim _{n \rightarrow \infty} z_{n}=0$. The claim follows.

For $\pi / 4<\theta$, we obtain $\tan \theta>1$. Let $\varepsilon>0$ such that $\tan \theta>(1+\varepsilon)^{2}$. By Theorem 2.7 there are natural numbers $n_{z}$ and $n_{\alpha}$ such that $(1+\varepsilon / 2) z_{n}>y_{n}$ for every $n>n_{z}$ and $(1+\varepsilon / 2) \sin \alpha_{n}>1$ for every $n>n_{\alpha}$. Set $n_{0}:=\max \left\{n_{z}, n_{\alpha}\right\}$. Then for every $n>n_{0}$, (2.1) implies

$$
\begin{aligned}
y_{n+1}^{2} & >\frac{1}{4} \cdot y_{n}^{2}+\frac{1}{4}(1+\varepsilon)^{4} \cdot z_{n}^{2}+(1+\varepsilon)^{2} \cdot \frac{1}{2} \sin \alpha_{n} \cdot y_{n} z_{n} \\
& >\frac{1}{4} \cdot y_{n}^{2}+\frac{1}{4}(1+\varepsilon)^{2} \cdot y_{n}^{2}+\frac{1}{2} \cdot y_{n}^{2} \\
& >\left(1+\frac{\varepsilon}{2}\right) y_{n}^{2} .
\end{aligned}
$$

Thus, $\lim _{n \rightarrow \infty} y_{n}=\infty$. Now $\lim _{n \rightarrow \infty} x_{n}=\infty$ follows from Theorem 2.7. 
For the last case, Corollary 2.2 implies $y_{n}=z_{n}$ for every $n>0$. The rest follows from (2.4).

We conclude this section with a statement concerning the orientation of the triangles $\Delta_{n}$.

Theorem 2.9. For every $n>0$, the triangle $\Delta_{n}$ is non-degenerate and counterclockwise oriented.

Proof. Assume that $\Delta_{n}$ is non-degenerate and counterclockwise oriented. By symmetric reasons we may assume $y_{n} \geq z_{n}$. Let $B_{n}^{\prime}$ and $C_{n}^{\prime}$ denote the centres of $C_{n} A_{n}$ and $A_{n} B_{n}$, respectively.

First we consider the case $x_{n} \geq y_{n}$. Then $\alpha_{n} \geq \beta_{n}$ and $\alpha_{n} \geq \gamma_{n}$ by the law of sines and therefore $\beta_{n} \leq \pi / 2$ and $\gamma_{n} \leq \pi / 2$. Since the triangle $A_{n+1} C_{n} B_{n}^{\prime}$ is similar to $\Delta_{n}$, we obtain $\angle C_{n} A_{n+1} B_{n}^{\prime}=\beta_{n}$. On the other hand, let $l$ be the perpendicular bisector of the side $y_{n}$, i.e. the line through $B_{n}^{\prime}$ and $B_{n+1}$. Since $x_{n} \geq z_{n}$, the line $l$ intersects $B_{n} C_{n}$ in a point $S$. We obtain $\angle C_{n} S B_{n+1}=\pi / 2-\gamma_{n}$. Thus, $\min \left\{\beta_{n}, \pi / 2-\gamma_{n}\right\} \leq$ $\angle C_{n} A_{n+1} B_{n+1} \leq \max \left\{\beta_{n}, \pi / 2-\gamma_{n}\right\}$ and therefore $0<\angle C_{n} A_{n+1} B_{n+1}<\pi / 2$. Analogously, $0<\angle C_{n+1} A_{n+1} B_{n}<\pi / 2$. This implies $\angle C_{n+1} A_{n+1} B_{n}<\pi$ and the claim holds for $\Delta_{n+1}$.

Now assume $x_{n}<y_{n}$. Then we obtain analogously to the above $0<\angle A_{n} B_{n}^{\prime} C_{n+1}<\pi / 2$ and $\angle A_{n+1} B_{n}^{\prime} C_{n}=\alpha_{n}<\pi / 2$. Thus, both angles $\angle A_{n+1} B_{n}^{\prime} B_{n+1}$ and $\angle B_{n+1} B_{n}^{\prime} C_{n+1}$ are greater than $\pi / 2$ and smaller than $\pi$ and consequently, $\angle C_{n+1} B_{n}^{\prime} A_{n+1}<\pi$. We conclude that $B_{n}^{\prime}$ is inside the triangle $\Delta_{n+1}$ and that the claim holds for $\Delta_{n+1}$.

We complete the proof by applying induction.

\section{Further remarks and outlook}

The "missing" case where only on one side, say $B_{n} C_{n}$, an isosceles triangle is erected and for the other two sides the centre is taken is not very interesting. Following I.M. Yaglom $\left[8\right.$, I.2, 22], the distinguished angle for the isosceles triangle would be $\angle C_{n} A_{n+1} B_{n}=0$ and therefore $\theta=\angle A_{n+1} B_{n} C_{n}=\angle B_{n} C_{n} A_{n+1}=\pi$, which is not possible. One gains the idea that the shape to which the triangles converge should be degenerate. Moreover, since every possible choice $\theta$ is smaller than $\pi$, the triangles should collapse to a single point. These claims are easy to prove: One can see immediately $x_{n+1}=x_{n} / 2$. Furthermore, $y_{n+1}<y_{n} / 2+\tan \theta \cdot x_{n} / 2$ and $z_{n+1}<z_{n} / 2+\tan \theta \cdot x_{n} / 2$. Thus, the ratios $x_{n} / y_{n}$ and $y_{n} / z_{n}$ tend to 0 while $y_{n} / z_{n}$ converges to 1 . After reaching the point $\tan \theta \cdot x_{n}<y_{n}$, we obtain additionally $y_{n+1}<y_{n}$. The analogue holds for $z_{n}$. Hence, $\lim _{n \rightarrow \infty} x_{n}=\lim _{n \rightarrow \infty} y_{n}=$ $\lim _{n \rightarrow \infty} z_{n}=0$.

Consequently, for triangles, the next task would be to consider three different angles $\theta_{x}, \theta_{y}$, and $\theta_{z}$ for the isosceles triangles that are erected on the sides of $\Delta_{n}$. Furthermore, instead of isosceles triangles one can erect arbitrary triangles on the sides of $\Delta_{n}$. One possibility to determine the triangles uniquely is to demand besides the angle $\theta$ (which we now ask for only one of the two possibilities) also the ratio $\lambda$ in which the side adjacent to the old triangle is subdivided by the orthocentre. This is in the spirit of [6] where the convergence of this transformation is studied by using its eigenvalues. 
Another possible generalisation would be to consider instead of the triangle $\Delta_{0}$ an arbitrary polygon. Of course, the transformation still uses isosceles triangles erected on the sides of the polygon. The convergence of these transformations has been studied in [5] together with the first case of this article. A possible application of this transformation is an element oriented mesh smoothing method based on successively applying this transformation to the polygonal bounded elements of triangular element surface meshes [4].

\section{References}

[1] Coxeter, H.S.M.; Greitzer, S.L.: Geometry revisited. The Mathematical Association of America: New Mathematical Library. Random House, New York 1967.

[2] Kiepert, L.: Solution de question 864. Nouv. Ann. Math. 8 (1869), 40-42.

[3] Rutherford, W.: VII. Quest. 1439. Ladies' Diary 122 (1825), 47.

[4] Vartziotis, D.; Athanasiadis, T.; Goudas, I.; Wipper, J.: Mesh smoothing using the geometric element transformation method. Comput. Methods Appl. Mech. Engrg. 197 (2008) 45-48, 3760-3767.

[5] Vartziotis, D.; Wipper, J.: Classification of symmetry generating polygon-transformations and geometric prime algorithms. Math. Pannon. 20 (2009) 2, 167-187.

[6] Vartziotis, D.; Wipper, J.: Characteristic parameter sets and limits of circulant Hermitian polygon transformations. Linear Algebra Appl. 433 (2010) 5, 945-955.

[7] Finsler, P.; Hadwiger, H.: Einige Relationen im Dreieck. Comment. Math. Helv. 10 (1937) 1, 316-326.

[8] Yaglom, I.M.: Geometric transformations. Random House, New York 1962. (Translated from the Russian by Allen Shields.)

\section{Dimitris Vartziotis}

Institute of Structural Analysis \& Antiseismic Research

National Technical University Athens (NTUA)

GR-15780 Athens, Greece

and

TWT GmbH Science \& Innovation, Research Department

Bernhäuser Straße 40-42

D-73765 Neuhausen, Germany

e-mail: dimitris.vartziotis@nikitec.gr

Simon Huggenberger

TWT GmbH Science \& Innovation, Research Department

Bernhäuser Straße 40-42

D-73765 Neuhausen, Germany

e-mail: simon.huggenberger@twt-gmbh.de 